\title{
Kombitherapie kriegt Schilddrüsenknoten klein
}

- Zur Behandlung von euthyreoten Knotenstrumen ist eine Kombination von Levothyroxin (T4) und lodid (I) besser geeignet als die Einzelkomponenten: Unter der Kombinationstherapie schrumpft das Volumen der Schilddrüsenknoten am stärksten. Das ist das Ergebnis der LISA-Studie, einer randomisierten Doppelblindstudie unter Beteiligung von 60 deutschen Studienzentren.

In der Studie wurden 1020 erwachsene Patienten mit euthyreoter Knotenstruma (Knotendurchmesser mindestens $1 \mathrm{~cm}$ ) einer von vier Behandlungsgruppen zugeteilt: T4 plus lodid, T4 allein, lodid allein oder Placebo. T4 wurde so (niedrig) dosiert, dass der TSH-Wert im Bereich von 0,2-0,8 mU/L lag.

Nach einem Jahr hatte das Gesamtvolumen der Knoten in allen drei Gruppen mit aktiver Therapie signifikant abgenommen: mit T4 + I um 21,6\%, mit T4 um 12,1\%, mit I um 9,0\% und mit Placebo um 5,2\% (nicht signifikant). Im Vergleich zu Placebo ergab sich eine Volumenreduktion um 17,3\% (T4 + I), 7,3\% (T4) und 4,0\% (I). Die Kombinationstherapie war damit signifikant wirksamer als die Monotherapien mit T4 oder I.

Die Knoten wurden unter T4 + I allmählicher

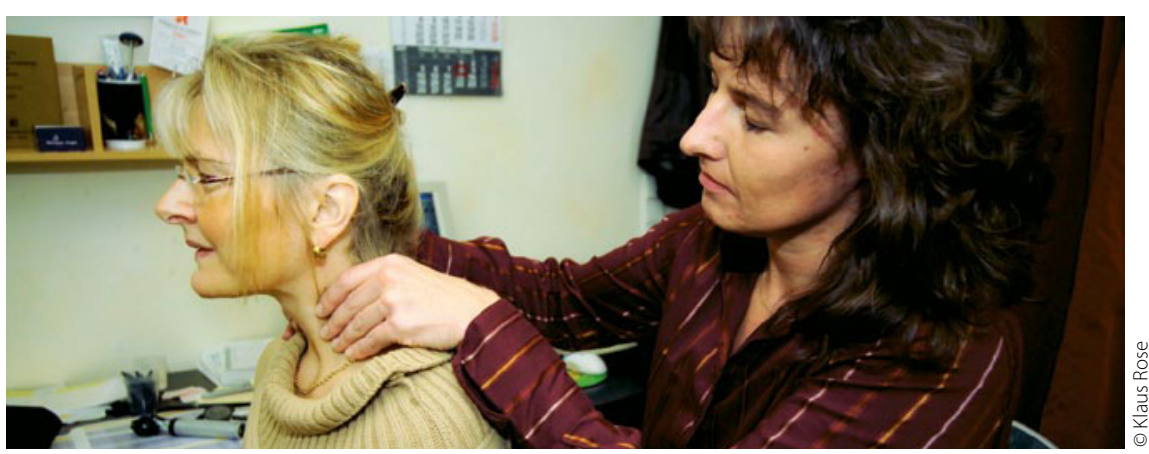

Die LISA-Studie zeigt, unter welcher Therapie die Struma am schnellsten schrumpft.

kleiner, und die Volumenabnahme hatte bei Studienende nach einem Jahr noch kein Plateau erreicht. Allerdings waren die Effekte der Therapie von Patient zu Patient verschieden. Bei $26,7 \%$ der Patienten unter T4 + I kam es zu einer Zunahme des Knotenvolumens.

Das Schilddrüsenvolumen wurde durch die aktiven Therapien ebenfalls reduziert: Im Vergleich zu Placebo um 7,9\% (T4 + I), $5,2 \%$ (T4) und 2,5\% (I). Bei Patienten mit großer Schilddrüse fiel auch die Abnahme des Schilddrüsenvolumens größer aus.

Das Fazit der Autoren um Prof. Martin Grussendorf, Stuttgart, lautet daher: „Patienten mit Schilddrüsenknoten sollten mit
T4 + I behandelt werden, um die Größe der Knoten zu begrenzen und neuen Knoten vorzubeugen."

BS =

Grussendorf M et al. Reduction of thyroid nodule volume by levothyroxine and iodine alone and in combination: a randomized, placebo-controlled trial. J Clin Endocrinol Metab, Sept 2011

\section{Veranstaltungs-Tipp}

Die „Therapie der diffusen und nodösen Struma" ist Thema des HenningSymposiums Schilddrüse 2011, das vom 29.9. bis 1.10.2011 in Heidelberg stattfindet. Anmeldung unter www. infoline-schilddruese.de

\section{OPTION FÜR LOW-RISK-PATIENTEN}

\section{Mehr Mut zur ambulanten Therapie von Lungenembolien}

\section{- Patienten mit Lungenembolie werden} meist stationär behandelt. Dabei gibt es unter ihnen viele Kandidaten, die mit ambulanter Versorgung nicht schlechter dran wären.

In einer multinationalen Studie waren Low-risk-Patienten mit akuter Lungenembolie randomisiert in zwei Gruppen eingeteilt worden. Die Patienten der Gruppe 1 $(n=171)$ wurden in der Selbstinjektion von Enoxaparin (1 mg/kg 2x/d) unterrichtet und innerhalb von 24 Stunden wieder aus der Klinik entlassen. Die Probanden der Gruppe $2(n=168)$ wurden stationär aufgenommen und erhielten ebenfalls eine
Enoxaparintherapie. Nach frühestens fünf Tagen wurde die Medikation in beiden Gruppen für mindestens weitere 90 Tage auf orale Vitamin-K-Antagonisten umgestellt. Alle Studienteilnehmer gehörten den Risikoklassen I oder II im „Pulmonary Embolism Severity Index" an.

Einer der 171 ambulant Behandelten erlitt ein Thromboembolie-Rezidiv; bei den stationären Patienten war kein solcher Fall zu verzeichnen. In beiden Gruppen verstarb je ein Patient innerhalb von 90 Tagen. Drei größere Blutungsereignisse binnen 90 Tagen ereigneten sich in Gruppe 1, keines in Gruppe 2. Die stationär aufgenommen Pa- tienten waren im Schnitt 3,9 Tage im Krankenhaus.

Das Resümee der Studienautoren fällt positiv aus: „Ausgewählte Patienten mit niedrigem Risiko, die eine Lungenembolie erlitten haben, können sicher und effektiv ambulant behandelt werden". Die ambulante Therapie werde von den Patienten gut akzeptiert und reduziere die Aufenthaltszeiten im Krankenhaus.

Aujesky D et al. Outpatient versus inpatient treatment for patients with acute pulmonary embolism an international, open label, randomized, noninferiority trial. Lancet 2011; doi: 10.1016/S01406736(11)60932-X. 